\title{
Patent Vitello - Intestinal Duct: A Case Report
}

\section{Bijay Thapa, Anupama Thapa and Anuj Kayasthya}

Department of Paediatric Surgery, Kanti Children's Hospital, Kathmandu, Nepal

\section{Correspondence:}

Bijay Thapa

Department of Paediatric Surgery,

Kanti Children's Hospital,

Kathmandu, Nepal

E-mail: bijaytapa@hotmail.com

DOI: $10.3126 /$ jnps.v41i1.30184

Submitted on: $2020-08-19$

Accepted on: 2021-02-14

Acknowledgements: None

Funding: Nil

Conflict of Interest: None declared

Permission from IRB: Yes

To cite this article: Thapa $\mathrm{B}$, Thapa A, Kayasthya A. Patent Vitello - Intestinal Duct: A Case Report. J Nepal Paediatr Soc. 2021;41(1):103-6.

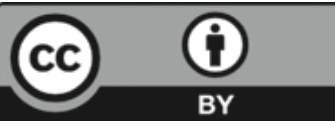

This work is licensed under creative common attribution 3.0 license

\section{ABSTRACT}

A wide variety of anomalies may occur as a result of the vitellointestinal duct (VID) failing to obliterate completely. These anomalies occur in approximately $2 \%$ of the population and may remain silent throughout life or may present incidentally with an intra-abdominal complication. Complete patency of vitello-intestinal duct is the rarest of all the anomalies of VID. We report a case of 27 day old female presenting with pinkish umbilical mass since birth referred to us by a paediatrician as umbilical granuloma, which turned out to be patent vitellointestinal duct.

Key words: granuloma; intestinal duct; vitellointestinal duct 


\section{INTRODUCTION}

Newborns and infants often present with a moist umbilicus which can be associated with various abnormalities from simple granulomas to persistent urachus. Discharge from umbilicus is a very common presentation and umbilical granuloma is the commonest differential diagnosis in such patients. Umbilical granuloma is commonly managed by chemical or electric cauterisation. Parents are often the first to notice this abnormality and see it as a pinkish mass around the umbilicus or a persistent serous discharge around the umbilicus soon after birth. ${ }^{1-5}$

When the lesion fails to respond to this treatment, patient should be further investigated for alternate diagnosis. Patent vitello-intestinal duct (VID) should be ruled out in such patients to avoid catastrophy. When a persistent serous, feculent or bilious drainage are identified at the umbilicus, it is suggestive of a patent VID with fistulous communication with the umbilicus. ${ }^{2,6-8}$ Treatment of patent VIDs requires surgical excision of the duct, with or without a segment of the small bowel, to obliterate the connection. Reconstruction of the umbilicus is then performed. ${ }^{9,10}$ The authors report a case of 27 day female neonate presenting with pinkish umbilical mass.

\section{CASE REPORT}

A 27 day old female neonate was referred for the evaluation of pinkish umbilical mass. She was born at term by normal vaginal delivery to a primigravida mother with birth weight of $3.5 \mathrm{~kg}$. At second week of life, mother noticed raised pinkish mass at the umbilicus with minimal serous discharge. The infant was otherwise clinically normal. The baby was managed locally with silver nitrate ointment and referred as the problem persisted.

On examination, a healthy infant with a pea-sized mass with a small opening was noted within the umbilicus with mild surrounding erythema (Figure 1). Some bilious discharge was also noted. A diagnosis of a patent VID was made clinically. A fistulogram through the umbilical opening using gastrograffin was performed and patent VID was confirmed (Figure 2).
The baby was managed with surgery on $38^{\text {th }}$ day of life. With a circumferential trans-umbilical skin incision, abdominal cavity was entered and the duct was successfully dissected from the abdominal wall. The duct connected to the small intestine was resected and closed. Umbilicoplasty was performed after closure of the muscular layer. Histology report of the duct showed intestinal structures without ectopic tissue. The postoperative course was uneventful and baby discharged and is asymptomatic on follow up.

\section{DISCUSSION}

At around third week of embryonic life, a communication exists between the embryonic gut and the yolk-sac which narrows into a tube called the VID, also called the omphalomesenteric duct (OMD). It usually gets obliterated by the end of the seventh week. In $2 \%$ of cases, persistence of various portions of VID gives rise to a spectrum of congenital anomalies like sinus, cyst, fistula, band and diverticulum. Meckel's diverticulum, vitelline cyst, vitelline ligament and umbilical sinus represent incomplete obliteration of the VID and are the most common forms of presentation. ${ }^{1,2}$

Patent VID is a result of complete failure of obliteration of this duct. The duct may remain patent throughout its course, producing an enterocutaneous fistula between the distal small intestine and the umbilicus. It is the rarest form among VID anomalies seen either in neonates or in infants with the incidence of $0.0063-0.067 \%{ }^{3}$ Exact etiology of incomplete obliteration is unknown. Different teratogenic models are

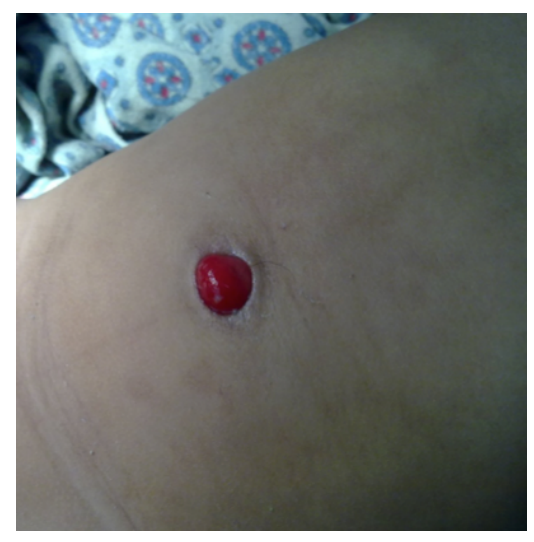

Figure 1. Umbilical mass 


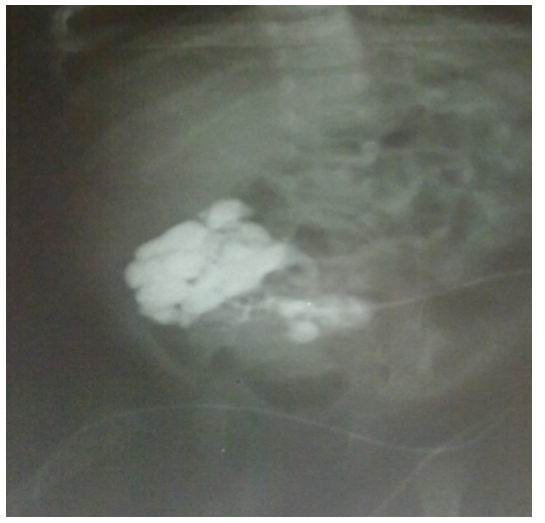

Figure 2. Fistulogram showing connection to small bowel

presented in the literature however none can accurately address a direct cause or effect relationship with these anomalies. In our patient, there was no history to explain such etiology. ${ }^{4}$

A patent VID can present as continued discharging umbilical sinus, umbilical nodule or polyp. The appearance and amount of the discharge can help make the diagnosis. If the discharge is persistently more and if it is bilious or yellowish in colour, then there may be associated cellulitis, which may progress to necrotising fascitis or sepsis. This was not seen in this case and only a small amount of bilious discharge was seen only on abdominal palpation. Patent VID is similar in presentation like umbilical granulomas which are benign lesions. Umbilical granuloma represents granulation tissue yet to epithelise. It is reddish in colour and bleed minimally when irritated by trauma. They are treated with cauterisation with $75 \%$ Silver Nitrate. If a granuloma fails to respond to such cauterisation, alternative diagnosis must be considered. Rarely granulomas need surgical removal. ${ }^{5}$ Ultrasound can be used to diagnose a suspected VID by identifying their relationship to and their continuity with the umbilicus and the urinary bladder. It also helps to prevent unnecessary surgical exploration and may guide for further management. ${ }^{6}$ The clinical diagnosis of this anomaly is sometimes simple and straight forward when fecal discharge is seen. Sometimes, fistulogram via the VID helps in confirming the diagnosis. $^{7}$

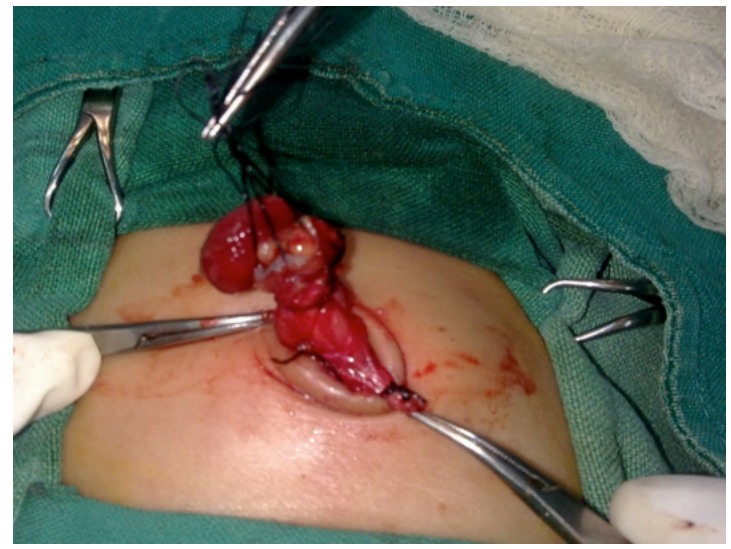

Figure 3. Trans-umbilical mobilization of VID

It is recommended that early surgical resection is the management. Its complications include omphalitis, bleeding from the protruded intestinal mucosa, prolapse and strangulation of the intestine and the tendency of malignancy. ${ }^{8}$

Different operative interventions have been described for the management. Surgery involves laparotomy to resect the connection to the intestine through a transverse infraumbilical incision, a transverse supraumbilical incision, a wide elliptic incision, or a laterally or vertically extended incision. We selected a trans-umbilical approach via a circular incision around the mucocutaneous junction, as recommended by Hagesawa $\mathrm{T}$ et al. followed by umbilicoplasty. ${ }^{9}$ The postoperative appearance was excellent. We had done few other such cases via infraumbilcal incision which had a large scar postoperatively. This is the first case report in Nepal managed with transumbilical approach. Laparoscopy has been attempted recently, but the insertion of at least two trocars was required with wide abdominal wall openings made larger wound than that in our approach. Therefore, the laparoscopic approach seems less advantageous than trans umbilical approach that we followed in our case. ${ }^{10}$

\section{CONCLUSIONS}

Patent VID should always be considered in the differential diagnosis of any case presenting with granulomatous umbilical lesion. Umbilical granuloma is common which responds well to silver nitrate but one should always consider other anomaly when it fails to respond. Therefore, careful 
examination of umbilicus is always necessary

before treatment. Trans-umbilical resection and

umbilicoplasty gives an excellent cosmetic result to patent VID.

\section{REFERENCES}

1. Agrawal S, Menon A. Patent vitellointestinal duct. BMJ Case Rep. 2010. DOI:10.1136/bcr.12.2009.2594

2. Zafer Y, Yigit S, Turken A. Patent omphalomesenteric duct. Turk J Med Sci. 2000; 30:83-85.

3. Rao PL, Mitra SK, Pathak IC. Patent vitello intestinal duct. Indian J Pediatr. 1979;46:215. DOI:10.1007/ BF02898231.

4. Frolov P, Alali J, Klein MD. Clinical risk factors for gastroschisis and omphalocele in humans: a review of the literature. Pediatr Surg Int. 2010;26:1135-48. DOI: 10.1007/s00383-010-2701-7

5. Piparsaliya S, Joshi M, Rajput N. Patent vitellointestinal duct: A close differential diagnosis of umbilical granuloma: A case re- port and review of literature. Surgical Science. 2011;2:134-6. DOI: 10.4236/ss.2011.23027

6. Khati NJ, Enquist EG, Javitt MC. Imaging of the umbilicus and periumbilical region. Radiographics. 1998; 18:413-31. DOI: 10.1148/radiographics.18.2.9536487

7. Maxwell D, Hariri N, Coleman KC. A case report of a patent omphalomesenteric duct presenting with meconium discharge from the umbilicus. Ann Clin Case Rep. 2016;1:2016;1022.

8. Zea MI, Chana RS, Anees A. Inverted ileal prolapse through patent vitellointestinal duct: a case report. Internet J PediatrNeonatol. 2009;10:2. DOI: https://doi.org/10.5580/28dc

9. Hasegawa T, Sakurai T, Monta O, Tazuke Y, Ueda S, Iwasaki Y, et al. Transumbilical resection and umbilical plasty for patent omphalomesenteric duct. Pediatr Surg Int. 1998;13:180-1. DOI: https://doi.org/10.1007/s003830050282

10. Lassen PM, Harris MJ, Kearse WS, Argueso LR. Laparoscopic management of incidentally noted omphalomesenteric mesenteric duct remnant. J Endourol. 8:49-51. DOI: 10.1089/end.1994.8.49. 